it is a map nonetheless (see, for example, NGA Nautical Chart 27).

A great deal of emphasis is freighted on the autonomy of points, but the primary value of points is not their independent existence as points, but their place in a topological context; a context represented by "a projection of a mental schema on a medium, the materialization of an abstract intellectual order extracted from the empirical universe" (Jacob 2006, 30). To wit: a place contextualized on an explicit or implicit map.

The author writes frequently of a geo-epistemology, which he finally defines (on page 298) as equivalent to geographic tools, but he seems to have a rather faulty grip on the ontology and epistemology of the map. It might seem necessary, if one is going to dismiss the relevance of the map, to engage with it; but, when Rankin gets close to doing so, he grandly dismisses the map as "representational," and thus an artifact of the past.

This sort of thing is what makes reading After the Map by turns so interesting, tedious, and infuriating: Rankin's persistent strong arming of a great deal of well-researched information into the straitjacket of his vaulting vision of the march of technology leaving the map behind for the utopia of a cloud of autonomous points.
Unfortunately for Dr. Rankin, the fact remains that the map has not been, and will not be, left behind: outmoded forms and practices are not "the map," they are just forms and practices. "The map" is an entirely artificial, cultural construct, and cartographic epistemology relies on a schema of mapicity that is mutable. The map, and its epistemology, is not tied to any particular form or style of artifact. While the map is ontologically stable as a useful, usable, and persuasive rhetorical artifact, its epistemology is culturally contingent. Maps can and will change: that is indisputable. That they are being left behind, as William Rankin contends in After the Map, remains, at best, unproven.

\section{REFERENCES}

House, Edward Mandell and Charles Seymour, eds. 1921. What Really Happened at Paris: The Story of the Peace Conference, 1918-1919. New York: Charles Scribner's Sons.

Jacob, Christian. 2006. The Sovereign Map. Translated by Tom Conley, edited by Edward Dahl. Chicago: University of Chicago Press.

Scahill, Jeremy. 2016. The Assassination Complex: Inside the Government's Secret Drone Warfare Program. New York: Simon and Schuster.

\title{
GETTING TO KNOW ARCGIS PRO
}

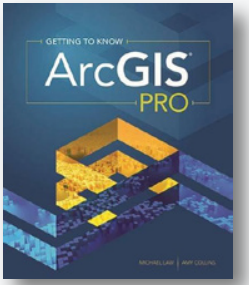

By Michael Law and Amy Collins.

Esri Press, 2016.

467 pages. \$85.99, softcover

ISBN: 978-1-58948-457-3

Review by: Tara LaLonde, The Pennsylvania State University

Getting to Know ArcGIS Pro provides the reader with ten well-structured chapters introducing features of ArcGIS Pro 1.1. The book includes background material on geographic information systems (GIS) principles, and the chapters build on one another in a manner appealing to both new and experienced GIS users, while at the same time incorporating new terminology specific to ArcGIS Pro. Sample data are provided by Esri via a companion website, for use with each chapter's exercises. Each of the text's exercises occurs in the context of a scenario related to a real-world problem or question. This project-based learning mirrors how GIS is taught in educational settings and how learners apply GIS concepts to new software and data.

Law and Collins guide readers through the application of practical and relevant GIS concepts in ArcGIS Pro through ten chapters. Each begins with an introduction, followed by a description of exercise datasets, exercise instructions, a summary, and a glossary of terms. This consistent framework and layout provides the reader with an easy to follow pattern as the material progresses from introductory GIS techniques to more advanced topics in later chapters. 
Chapter 1, "Introducing GIS," provides an introduction to basic GIS principles of vectors, layers, rasters, and attributes, along with an exercise in ArcGIS Online. The exercise presents the reader with opportunity to explore ArcGIS Online through an organizational account, offering an opportunity to understand how an ArcGIS Online organizational account works in relation to ArcGIS Pro. The text points readers to the State of Michigan's GIS data resources, providing them with an example of an open data site, which are becoming increasingly common. Many additional data sources and websites are presented, enhancing the value of the book as a guide to learning ArcGIS Pro and GIS principles.

To provide a foundation in ArcGIS Pro terminology, Chapter 2, "A First Look at ArcGIS Pro," introduces the reader to key terms unique to the software, such as a project files and project packages, along with ways for sharing data and files with collaborators and other users. The tabs-and-panel structure of the software is presented in an easy to read layout, with examples that could relate to many projects across disciplines. To prepare the reader for navigating later exercises, this chapter describes the folder structures of the exercise data and associated files within geodatabases, and also introduces the reader to an engaging feature of ArcGIS Pro: the application's side-by-side view of $2 \mathrm{D}$ and $3 \mathrm{D}$.

In subsequent chapters, readers build on this foundation of working with spatial data, but are also provided helpful tips and reminders of earlier chapters to reinforce material already covered. In Chapter 3, "Exploring Geospatial Relationships," the reader works through the process of joining data and creating an index. Helpful descriptions of the different symbology classifications are provided to aid the user in selecting the most appropriate classification scheme. A key feature presented in this exercise is the ability to swipe across layers, which provides the user with unique comparison opportunities. The concept of multiple map tabs is also introduced, allowing the user to have multiple maps as part of the same project file. Finally, key terminology related to feature classes, layer files, and layer packages provide context to the use of these files in exercises. Readers use this solid foundation as they next work through an introduction to geodatabases, geoprocessing tools, and editing spatial data in Chapter 4, "Creating and Editing Spatial Data." This chapter also introduces the reader to working with $\mathrm{XY}$ data, and the concept of metadata; however, additional material on metadata could have further conveyed its importance in identifying dataset characteristics.

In Chapter 5, "Facilitating Workflows," the reader is introduced to ModelBuilder and working with Python scripts. Users are guided through the process of creating their own tasks, which is a new concept in the ArcGIS Pro software. Pre-configured tasks are used to walk the user through the different features of creating and describing a task, which can then be packaged and shared so that others can repeat them; this automation is a key part of the creation of tasks. The emphasis on making collaboration easier is followed by Chapter 6, "Collaborative Mapping," which focuses on collaborative mapping fieldwork through the creation of a tree inventory geodatabase. The reader is given additional experience with the use of geodatabase domains and properties, along with the connections to Collector for ArcGIS. They also learn about publishing map layers to ArcGIS Online, which further demonstrates how layers can be shared between ArcGIS Pro and ArcGIS Online.

Once they have a grasp of GIS foundations, navigating the ArcGIS Pro panels and layout, and the collaborative possibilities available, readers gain more advanced experience with geospatial analysis in the last four chapters. In Chapter 7, "Geoenabling Your Project," the reader is exposed to the concepts of geocoding and creating buffers, which are analysis techniques they may find useful in a variety of later contexts. This is followed by a crime scenario in Chapter 8, "Analyzing Spatial and Temporal Patterns." Here, the user examines data across time and space using the kernel density function, hot spot analysis, and a spacetime cube. The exercise's 3D scenes demonstrate ArcGIS Pro's 3D capabilities and potential. In addition to presenting new software functions in each chapter, different geospatial file formats are also incorporated in the exercises, such as the netCDF (network Common Data Form) format for multidimensional data.

The path toward more advanced GIS concepts continues through a vineyard suitability example in Chapter 9, "Determining Suitability." This scenario exposes the reader to elevation models, hillshade, slope, and aspect, reclassification, the Model Builder, and the raster calculator. This scenario demonstrates how raster files are analyzed in ArcGIS Pro and teaches skills that relate to other suitability tasks the reader may eventually have. The text also points readers toward a valuable, web-based resource for determining the altitude and azimuth of the sun at a 
given location for any given time and date, which can be used to determine hillshade parameters. Finally, following this suitability analysis, the text takes readers through the software's presentation options in Chapter 10, "Presenting Your Project.” An example related to mapping broadband service providers is used to explain to readers how to display a subset of a layer's features, label locations, and lets them explore page layout and export options. This page layout section in particular offers a good overview of features that are crucial to communicating results from geospatial analysis in ArcGIS Pro.

This book serves as a valuable resource for GIS users desiring to learn more about the ArcGIS Pro application, educators incorporating ArcGIS Pro into curricula, libraries providing software access to patrons, and individuals interested in exploring GIS software for the first time. It presents many of the common tasks users perform to manipulate and analyze geospatial information. The scenario exercises include a range of subject areas including population, conflict, broadband access, and demographics, providing the reader with many real-world applications involving geospatial data; these could be adopted by educators and tailored to specific course curricula. The current edition of the book includes a limited number of scenarios, allowing students and educators to complete the book in a realistic timeframe. However, it would be helpful in future editions to see exercise scenarios extended to additional geographic areas and application fields, in order to appeal to a broader audience of users. Overall, the book achieves its aims in offering a good introduction to ArcGIS Pro, as well as providing readers with a foundational understanding of how to work with geospatial data that they can apply to additional GIS resources, learning opportunities, and workplace environments.

\section{THE CURIOUS MAP BOOK}

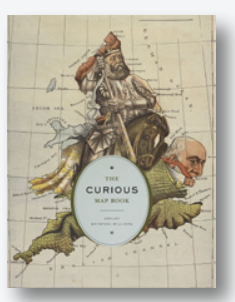

by Ashley Baynton-Williams

University of Chicago Press, 2015

240 pages, 100 color plates. $\$ 45.00$, hardcover.

ISBN: 978-0-226-23715-2

\section{Review by: Mark Denil}

Cartographic curiosities are always engaging, as demonstrated by the variety of map miscellanies available on the market. The Curious Map Book by Ashley BayntonWilliams might, at first glance, seem to be just another such compendium of a few odds and sods tossed together to milk the trade; but that impression would be wrong. It is, in fact, an excellent, long-baseline overview of unique and interesting cartographic curiosities assembled by someone with a comprehensive grasp of the historical horizon.

The layout of the book is familiar enough: two columns of descriptive text on the left page and a full-map, full-color illustration on the right. Although the author, BayntonWilliams, "is an antiquarian map dealer and researcher," his commentary is equally far from both dreary pedantry and salesroom sophistry. His remarks are short and concise, with enough description to illuminate the exhibit and enough context to establish the map's position in history. The accompanying photos are clear and sharp, and provide a reasonably good view of the map under consideration. The main photo pages are supplemented with twenty-two additional detail images (on the appropriate description pages) and five two-page photo spreads of particularly large and detailed maps. The maps with two-page spreads usually use their regular illustration page for a full-bleed detail shot.

The volume itself is solidly bound, with pages that fall open easily and sit between boards covered with green cloth. The dust cover has fine, large, illustrations front and back, and has a spine title block that mimics a pasted label. The book has a nice heft, and is sized to balance well in the hand. The paper has a medium gloss, and, while it is not particularly opaque, the book design makes that less of a problem than it might be. The left-hand pages have lots of clear white space with sharp black text, while the right-hand pages (that are, in practice, the back sides of the left-hand pages) are covered with solid, full-bleed ink: the parts not covered by the map are filled with a dense background color that varies by book section. This helps to give the white page areas on the reverse a good, smooth, non-distracting ground; this is an effect that may or may not have been in the mind of the book designer, but which is appreciated. 